\title{
Generalized Exponential Distribution: BAYESIAN EsTIMATIONS
}

\author{
Debasis Kundu ${ }^{1}$ and Rameshwar D. Gupta ${ }^{2}$
}

\begin{abstract}
Recently two-parameter generalized exponential distribution has been introduced by the authors. In this paper we consider the Bayes estimators of the unknown parameters under the assumptions of gamma priors on both the shape and scale parameters. The Bayes estimators can not be obtained in explicit forms. Approximate Bayes estimators are computed using the idea of Lindley. We also propose Gibbs sampling procedure to generate samples from the posterior distributions and in turn computing the Bayes estimators. The approximate Bayes estimators obtained under the assumptions of non-informative priors, are compared with the maximum likelihood estimators using Monte Carlo simulations. One real data set has been analyzed for illustrative purposes.
\end{abstract}

Key Words and Phrases: Bayes estimator; Maximum likelihood estimator; Gamma distribution; Log-concave density function; Squared error loss function; Posterior density function.

Address of correspondence: Debasis Kundu, e-mail: kundu@iitk.ac.in, Phone: 91-5122597141, Fax: 91-512-2597500.

${ }^{1}$ Department of Mathematics and Statistics, Indian Institute of Technology Kanpur, Pin 208016, India.

${ }^{2}$ Department of Computer Science and Applied Statistics. The University of New Brunswick, Saint John, Canada, E2L 4L5. Part of the work was supported by a grant from the Natural Sciences and Engineering Research Council. 


\section{INTRODUCTION}

Recently the authors proposed [4] the two-parameter generalized exponential distribution (GE) as an alternative to the gamma and Weibull distributions and studied its different

properties. Some of the recent references on GE distribution are Raqab [16], Raqab and Ahsanullah [17], Zheng [23], Sarhan [20], Gupta and Kundu [6, 8] and the references cited there.

The two-parameter GE has the following density function

$$
f(x ; \alpha, \lambda)=\alpha \lambda\left(1-e^{-\lambda x}\right)^{\alpha-1} e^{-\lambda x} ; \quad x>0
$$

here $\alpha, \lambda>0$ are the shape and scale parameters respectively. The main aim of this paper is to consider the Bayesian analysis of the GE distribution and compare their performances with the classical ones. Since both $\alpha$ and $\lambda$ are non-negative therefore, it is quite natural to assume the gamma priors on $\alpha$ and $\lambda$, although they are not the conjugate priors. In many practical situations, the information about the shape and scale of the sampling distribution is available in an independent manner, see Basu, Basu and Mukhopadhyay [2]. Therefore, here it is assumed that the parameters $\alpha$ and $\lambda$ are independent a priori.

In this paper we mainly consider the squared error loss function. It is observed that the Bayes estimators can not be expressed in explicit forms and they can be obtained by two dimensional numerical integrations only. We use the idea of Lindley to compute the approximate Bayes estimators of the unknown parameters and it is observed that the approximation works quite well. We compute the approximate Bayes estimators under the assumption of non-informative priors and compare them with the maximum likelihood estimators (MLEs) by Monte Carlo simulations. We also propose Markov Chain Monte Carlo (MCMC) techniques to generate samples from the posterior distributions and in turn computing the Bayes estimators. The posterior density functions match quite well with the histograms of the 
samples obtained by MCMC methods.

It should be mentioned that Jaheen [7] and Raqab and Madi [18] also considered the Bayesian inferences of the unknown parameter(s) of the GE distribution. Jaheen [7] considered the empirical Bayes estimate of the shape parameter when the scale parameter is known. Raqab and Madi [18] considered the case when both the parameters are unknown, but their approaches and the emphasis are quite different than the present paper. Moreover, in this paper we have observed that our method can be extended to a more general class of distributions, for example proportional reversed hazard models or for exponentiated Weibull distribution. However, it is not clear how to extend the results of Jaheen [7] and Raqab and Madi [18] in a more general situation.

The rest of the paper is organized as follows. In section 2, we propose the Bayes estimators of the unknown parameters. The approximate Bayes estimators are also considered in section 2. One data set has been analyzed in section 3. Numerical results are provided in section

4. In section 5 we briefly mentioned how to extend our results for more general classes of distributions and finally conclusions appear in section 6 .

\section{Bayes Estimation of the Unknown Parameter(s)}

In this section we consider the Bayes estimation of the unknown parameter(s). When both are unknown, it is assumed that $\alpha$ and $\lambda$ have the following gamma prior distributions;

$$
\begin{aligned}
& \pi_{1}(\lambda) \propto \lambda^{b-1} e^{-a \lambda} ; \quad \lambda>0, \\
& \pi_{2}(\alpha) \propto \alpha^{d-1} e^{-c \alpha} ; \quad \alpha>0 .
\end{aligned}
$$

Here all the hyper parameters $a, b, c, d$ are assumed to be known and non-negative.

Suppose $\left\{x_{1}, \ldots, x_{n}\right\}$ is a random sample from $G E(\alpha, \lambda)$, then based on the likelihood 
function of the observed data;

$$
l(\operatorname{data} \mid \alpha, \lambda)=\alpha^{n} \lambda^{n} e^{-\lambda \sum_{i=1}^{n} x_{i}} \prod_{i=1}^{n}\left(1-e^{-\lambda x_{i}}\right)^{\alpha-1},
$$

the joint posterior density function of $\alpha$ and $\lambda$ can be written as;

$$
l(\alpha, \lambda \mid d a t a)=\frac{l(\text { data } \mid \alpha, \lambda) \pi_{1}(\lambda) \pi_{2}(\alpha)}{\int_{0}^{\infty} \int_{0}^{\infty} l(\operatorname{data} \mid \alpha, \lambda) \pi_{1}(\lambda) \pi_{2}(\alpha) d \alpha d \lambda}
$$

Therefore, the Bayes estimator of any function of $\alpha$ and $\lambda$, say $g(\alpha, \lambda)$ under the squared error loss function is

$$
\hat{g}_{B}=E_{\alpha, \lambda \mid \text { data }}(g(\alpha, \lambda))=\frac{\int_{0}^{\infty} \int_{0}^{\infty} g(\alpha, \lambda) l(\text { data } \mid \alpha, \lambda) \pi_{1}(\lambda) \pi_{2}(\alpha) d \alpha d \lambda}{\int_{0}^{\infty} \int_{0}^{\infty} l(\text { data } \mid \alpha, \lambda) \pi_{1}(\lambda) \pi_{2}(\alpha) d \alpha d \lambda} .
$$

It is not possible to compute analytically (6) in this case. Two approaches are used here namely (a) Lindley's approximation; (b) Markov Chain Monte Carlo (MCMC) to approximate (6). Note that when one parameter is known, the Bayes estimator of any function of the other parameter also can be written similarly as the ratio of two integrals such as (6).

\subsection{LindLEy's ApPROXimation}

Lindley [11] proposed his procedure to approximate the ratio of the two integrals such as

(6). This has been used by several authors to obtain the approximate Bayes estimators. For details see Lindley [11] or Press [14]. Based on Lindley's approximation, the approximate Bayes estimators of $\alpha$ and $\lambda$ under the squared errors loss function are

$$
\begin{aligned}
\hat{\alpha}_{B}= & \hat{\alpha}+\frac{1}{2}\left[\frac{2 n}{\hat{\alpha}^{3}} \tau_{11}^{2}+\left(\frac{2 n}{\hat{\lambda}^{3}}+(\hat{\alpha}-1) \sum_{i=1}^{n} \frac{x_{i}^{3} e^{-\hat{\lambda} x_{i}}\left(1+e^{-\hat{\lambda} x_{i}}\right)}{\left(1-e^{-\hat{\lambda} x_{i}}\right)^{3}}\right) \tau_{21} \tau_{22}\right. \\
& \left.-\left(\sum_{i=1}^{n} \frac{x_{i}^{2} e^{-\hat{\lambda} x_{i}}}{\left(1-e^{-\hat{\lambda} x_{i}}\right)^{2}}\right)\left(\tau_{22} \tau_{11}+2 \tau_{21}^{2}\right)\right]+\left(\frac{d-1}{\hat{\alpha}}-c\right) \tau_{11}+\left(\frac{b-1}{\hat{\lambda}}-a\right) \tau_{12}, \\
\hat{\lambda}_{B}= & \hat{\lambda}+\frac{1}{2}\left[\frac{2 n}{\hat{\alpha}^{3}} \tau_{12} \tau_{11}+\left(\frac{2 n}{\hat{\lambda}^{3}}+(\hat{\alpha}-1) \sum_{i=1}^{n} \frac{x_{i}^{3} e^{-\hat{\lambda} x_{i}}\left(1+e^{-\hat{\lambda} x_{i}}\right)}{\left(1-e^{-\hat{\lambda} x_{i}}\right)^{3}}\right) \tau_{22}^{2}\right.
\end{aligned}
$$




$$
\left.-\left(\sum_{i=1}^{n} \frac{3 x_{i}^{2} e^{-\hat{\lambda} x_{i}}}{\left(1-e^{-\hat{\lambda} x_{i}}\right)^{2}}\right)\left(\tau_{22} \tau_{21}\right)\right]+\left(\frac{d-1}{\hat{\alpha}}-c\right) \tau_{21}+\left(\frac{b-1}{\hat{\lambda}}-a\right) \tau_{22}
$$

respectively, here

$$
\begin{gathered}
\tau_{11}=\frac{W}{U W-V^{2}}, \quad \tau_{12}=-\frac{V}{U W-V^{2}}=\tau_{21}, \quad \tau_{22}=\frac{U}{U W-V^{2}} \quad \text { and } \\
U=\frac{n}{\hat{\alpha}^{2}}, \quad V=-\sum_{i=1}^{n} \frac{x_{i} e^{-\hat{\lambda} x_{i}}}{\left(1-e^{-\hat{\lambda} x_{i}}\right)}, \quad W=\frac{n}{\hat{\lambda}^{2}}+(\hat{\alpha}-1) \sum_{i=1}^{n} \frac{x_{i}^{2} e^{-\hat{\lambda} x_{i}}}{\left(1-e^{-\hat{\lambda} x_{i}}\right)^{2}} .
\end{gathered}
$$

For the proof of (7) and (8) see in the appendix.

If $\alpha$ is known, under the same assumption, as given in (2), of prior on $\lambda$, the Bayes estimator of $\lambda$, can not be expressed in explicit form. The approximate Bayes estimator of $\lambda$ under the squared error loss function is

$$
\begin{aligned}
\hat{\lambda}_{B}= & \hat{\lambda}+\frac{1}{n}\left[((b-1) \ln \hat{\lambda}-a \hat{\lambda})\left(\frac{1}{\hat{\lambda}^{2}}+(\alpha-1) \frac{1}{n} \sum_{i=1}^{n} \frac{x^{2} e^{-\hat{\lambda} x_{i}}}{\left(1-e^{-\hat{\lambda} x_{i}}\right)^{2}}\right)^{-1}+\right. \\
& \left.\frac{1}{4}\left(\frac{1}{\hat{\lambda}^{2}}+(\alpha-1) \frac{1}{n} \sum_{i=1}^{n} \frac{x^{2} e^{-\hat{\lambda} x_{i}}}{\left(1-e^{-\hat{\lambda} x_{i}}\right)^{2}}\right)^{-2} \times\left(\frac{2}{\hat{\lambda}^{3}}+(\alpha-1) \frac{1}{n} \sum_{i=1}^{n} \frac{x_{i}^{3}\left(1+e^{-\hat{\lambda} x_{i}}\right)}{\left(1-e^{-\hat{\lambda} x_{i}}\right)^{3}}\right)\right] .
\end{aligned}
$$

When $\lambda$ is known, under the same assumption of prior on $\alpha$ as given in (3), the posterior density of $\alpha$ is gamma with the shape and scale parameters as $d+n$ and $c-\sum_{i=1}^{n} \ln \left(1-e^{-\lambda x_{i}}\right)$ respectively. Therefore, under the square error loss function the Bayes estimator of $\alpha$ is

$$
\frac{n+d}{c-\sum_{i=1}^{n} \ln \left(1-e^{-\lambda x_{i}}\right)}
$$

Note that, as expected, for the non-informative prior, i.e. when $c=d=0$, the Bayes estimator and MLE of $\alpha$ are identical.

\subsection{MCMC Method}

In this subsection, we consider the MCMC method to generate samples from the posterior distributions and then compute the Bayes estimators of $\alpha$ and $\lambda$ under the squared errors 
loss function. Note that once we have the MCMC samples, it is possible to compute the Bayes estimators with respect to any other loss function also.

The joint posterior density function of $\alpha$ and $\lambda$ can be written as

$$
l(\alpha, \lambda \mid \text { data }) \propto \alpha^{n+d-1} \lambda^{n+b-1} e^{-\lambda\left(a+\sum_{i=1}^{n} x_{i}\right)} e^{-c \alpha} \prod_{i=1}^{n}\left(1-e^{-\lambda x_{i}}\right)^{\alpha-1},
$$

from (11) it is clear that the posterior density function of $\alpha$ given $\lambda$ is

$$
l(\alpha \mid \lambda, d a t a) \propto \alpha^{n+d-1} e^{-\alpha\left(c-\sum_{i=1}^{n} \ln \left(1-e^{-\lambda x_{i}}\right)\right)} .
$$

Therefore, the posterior density function of $\alpha$ given $\lambda$ is gamma with the shape and scale parameters as $n+d$ and $\left(c-\sum_{i=1}^{n} \ln \left(1-e^{-\lambda x_{i}}\right)\right)$ respectively.

Now let us also compute the posterior density function of $\lambda$ given $\alpha$. The posterior density function of $\lambda$ given $\alpha$ can be written as

$$
l(\lambda \mid \alpha, \text { data }) \propto \lambda^{n+b-1} e^{-\lambda\left(a+\sum_{i=1}^{n} x_{i}\right)} \prod_{i=1}^{n}\left(1-e^{-\lambda x_{i}}\right)^{\alpha-1} .
$$

From, $\frac{\partial^{2} \ln l(\lambda \mid \alpha, \text { data })}{\partial \lambda^{2}}$, it is immediate that $l(\lambda \mid \alpha$, data $)$ is $\log$-concave for $\alpha \geq 1$. For $\alpha \geq 1$, we use the general method of Devroye [3] to generate random sample from the log-concave density function $l(\lambda \mid \alpha$, data $)$.

For $\alpha<1$, generation from (13) needs special attention. For $\alpha<1$, let us rewrite (13) as

$$
l(\lambda \mid \alpha, \text { data }) \propto\left(1-e^{-\lambda x_{(1)}}\right)^{\alpha-1} e^{-\lambda x_{(1)}}\left[e^{-\lambda\left(a+\sum_{i=2}^{n} x_{(i)}\right)} \lambda^{n+b-1} \prod_{i=2}^{n}\left(1-e^{-\lambda x_{(i)}}\right)^{\alpha-1}\right]
$$

here $x_{(1)}<\ldots x_{(n)}$ are the ordered $x_{i}$ 's. Observe that the function within [.] is a continuous function. It goes to a finite constant as $\lambda$ approaches 0 and goes to 0 as $\lambda$ approaches $\infty$. It implies that the function has a finite maximum. Therefore with the help of the acceptance rejection principle (see Devroye [3] for details), the generation from (14) can be performed using the GE generator. 
Note that when $a=b=c=d=0$, then the priors are not proper, but even in that case the conditional posterior distributions, namely $l(\alpha \mid \lambda$, data $)$ and $l(\lambda \mid \alpha$, data $)$ are proper. We propose the following MCMC procedure.

MCMC METHOD:

- Step 1: Take some initial guess of $\alpha$ and $\lambda$, say $\alpha_{0}$ and $\lambda_{0}$ respectively.

- Step 2: Suppose at the $i-t h$ step, $\alpha$ and $\lambda$ take the values $\alpha_{i}$ and $\lambda_{i}$, then we generate $\alpha_{i+1}$ and $\lambda_{i+1}$ from $l\left(\alpha \mid \lambda_{i}\right.$, data $)$ and $l\left(\lambda \mid \alpha_{i}\right.$, data $)$ respectively.

- Step 3: Repeat Step 2, $N$ times.

- Step 4: Calculate Bayes estimator of $g(\alpha, \lambda)$ by

$$
\frac{1}{N-M} \sum_{i=M+1}^{N} g\left(\alpha_{i}, \lambda_{i}\right)
$$

where $M$ is the burn-in period.

Now note that when $\lambda$ is known the posterior density function of $\alpha$ can be written as (12) and similarly, the posterior density function of $\lambda$ can be written as (13). Therefore, posterior samples from the corresponding density functions can be drawn as mentioned above and the corresponding MCMC methods can be easily developed.

\section{Data Analysis}

In this section we analyze one data set and demonstrate how the proposed methods can be used in practice. The data (Lawless [9]; page 228) presented here arose in tests on endurance of deep groove ball bearings. The data presented are the number of million revolution before failure for each of the 23 ball bearings in the life test and they are; 17.88, 28.92, 33.00, 41.52, 
$42.12,45.60,48.80,51.84,51.96,54.12,55.56,67.80,68.64,68.64,68.88,84.12,93.12,98.64$, 105.12, 105.84, 127.92, 128.04 and 173.40.

We analyze the data using the generalized exponential distribution. It is known [4] that the MLE of $\alpha$ can be written as

$$
\hat{\alpha}(\lambda)=-\frac{n}{\sum_{i=1}^{n} \ln \left(1-e^{-\lambda x_{i}}\right)} .
$$

Therefore, the profile log-likelihood function (say $h(\lambda))$ of $\lambda$ becomes

$$
h(\lambda)=n \ln \hat{\alpha}(\lambda)+n \ln \lambda-\lambda \sum_{i=1}^{n} x_{i}-\sum_{i=1}^{n} \ln \left(1-e^{-\lambda x_{i}}\right) .
$$

We provide the profile log-likelihood function of $\lambda$ in Figure 1. Using the simple iterative

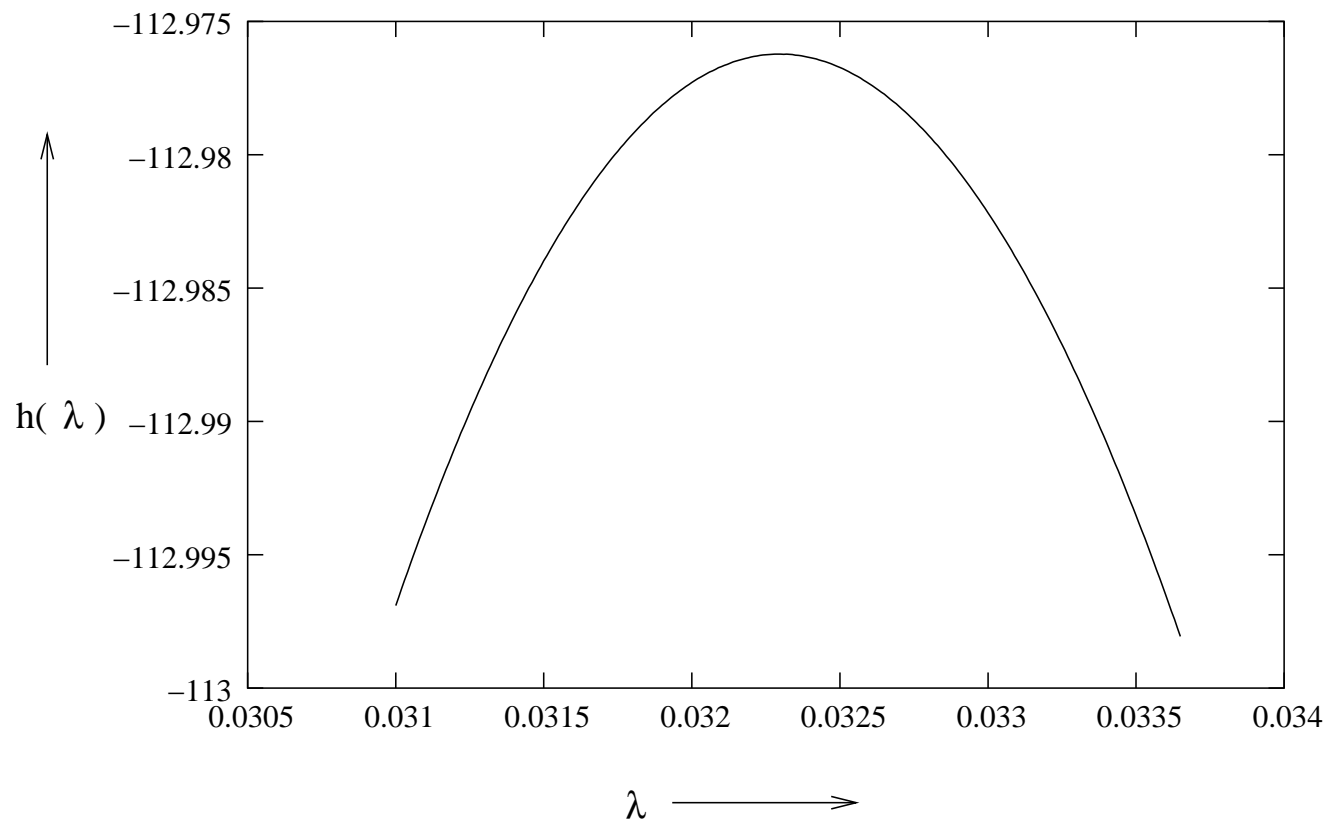

Figure 1: Profile log-likelihood function of $\lambda$.

procedure as suggested in Gupta and Kundu [5], the MLEs of $\alpha$ and $\lambda$ can be obtained as 5.2836 and 0.0323 respectively.

Now we compute the Bayes estimators of $\alpha$ and $\lambda$. Since we do not have any prior information, we assume $a=b=c=d=0$. Although it implies improper priors on $\alpha$ and 
$\lambda$, but the corresponding posteriors are proper. We compute the exact Bayes estimates of $\alpha$ and $\lambda$ under the squared error loss functions using numerical integration and they are 5.3466 and 0.0318 respectively. We compute the approximate Bayes estimates of $\alpha$ and $\lambda$ using Lindley's approximation and they are 5.3482 and 0.0318. It is interesting that Lindley's approximation works very well in this case.

We also compute the approximate Bayes estimates of $\alpha$ and $\lambda$ using MCMC method and they are 5.1287 and 0.0311 respectively. We plot the histograms of the samples of $\alpha$ and $\lambda$ generated by MCMC method along with their exact posterior density functions obtained numerically, in Figures 2 and 3 respectively.

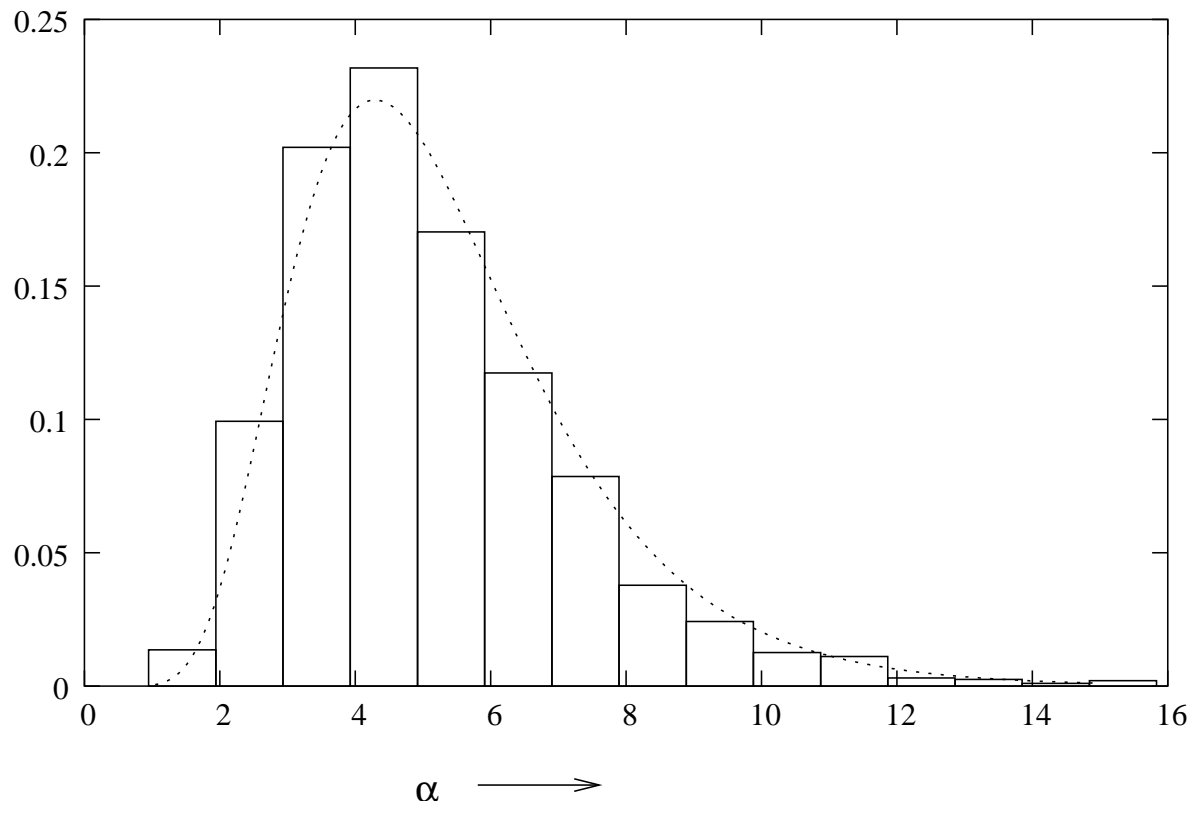

Figure 2: Posterior density function of $\alpha$ and the histogram of $\alpha$ generated by MCMC method.

From the Figures 2 and 3 it is clear that the exact posterior density functions match quite well with the simulated samples obtained using MCMC method. Therefore, MCMC samples can be used for constructing the approximate credible intervals or for example estimating any functions of parameters also quite effectively, they are not pursued here. 


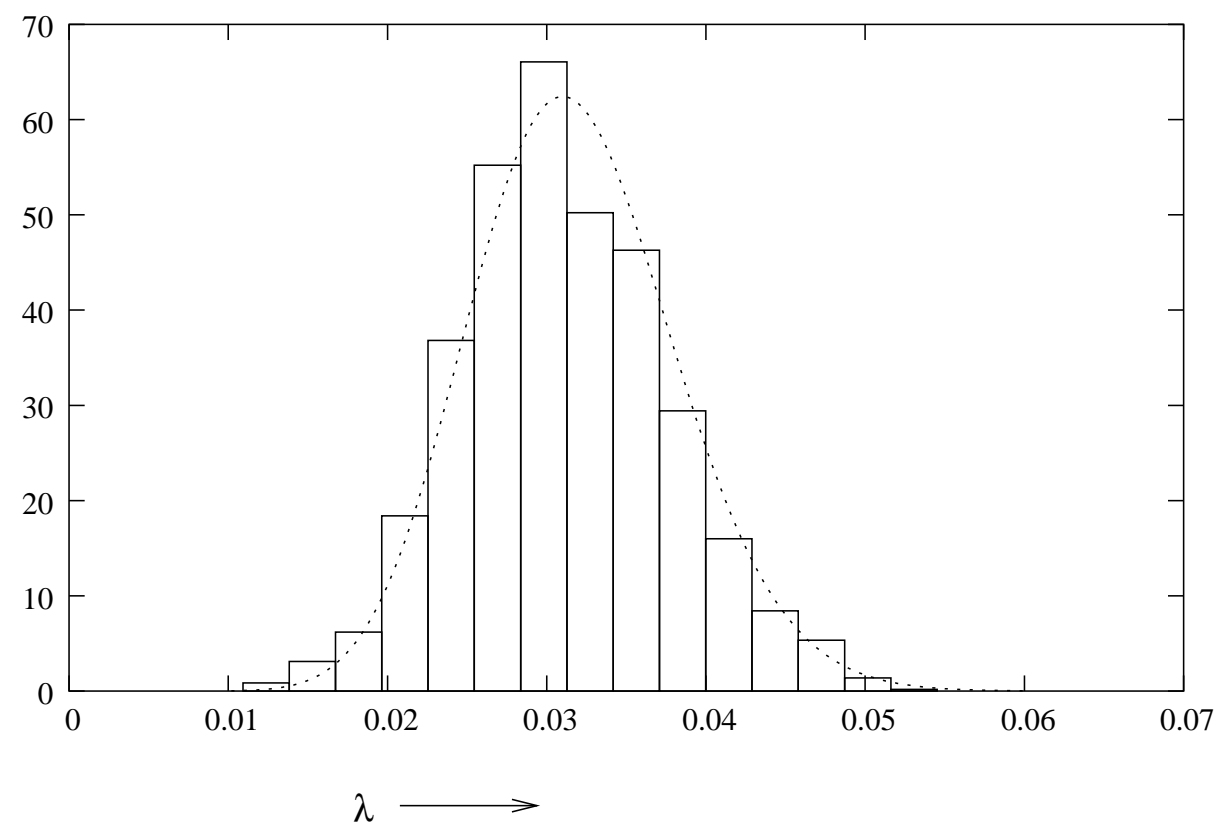

Figure 3: Posterior density function of $\lambda$ and the histogram of $\lambda$ generated by MCMC method.

It appears at least, that the Bayes estimate of $\alpha$ obtained using MCMC method (5.1287) is significantly different than the exact Bayes estimate of $\alpha$ (5.3466). We plot the density functions $f(x ; 5.3466,0.0318)$ and $f(x ; 5.1287,0.0311)$ in Figure 4 . Although the Bayes estimates are different but the estimated density functions obtained using them are almost indistinguishable.

To check the validity of the model, we compute the Kolmogorov-Smirnov (KS) distance between the empirical distribution function and the fitted distribution functions when the parameters are obtained by different methods, namely by MLEs, exact Bayes estimators and approximate Bayes estimators (MCMC). The results are presented in Table 1. From Table 1 it is clear that the estimated GE distributions provide excellent good fit to the given data. We plot the empirical survival function and the estimated survival functions obtained using the MLEs, exact Bayes and approximate Bayes in Figure 5. It shows that all of them fit the data very well. 


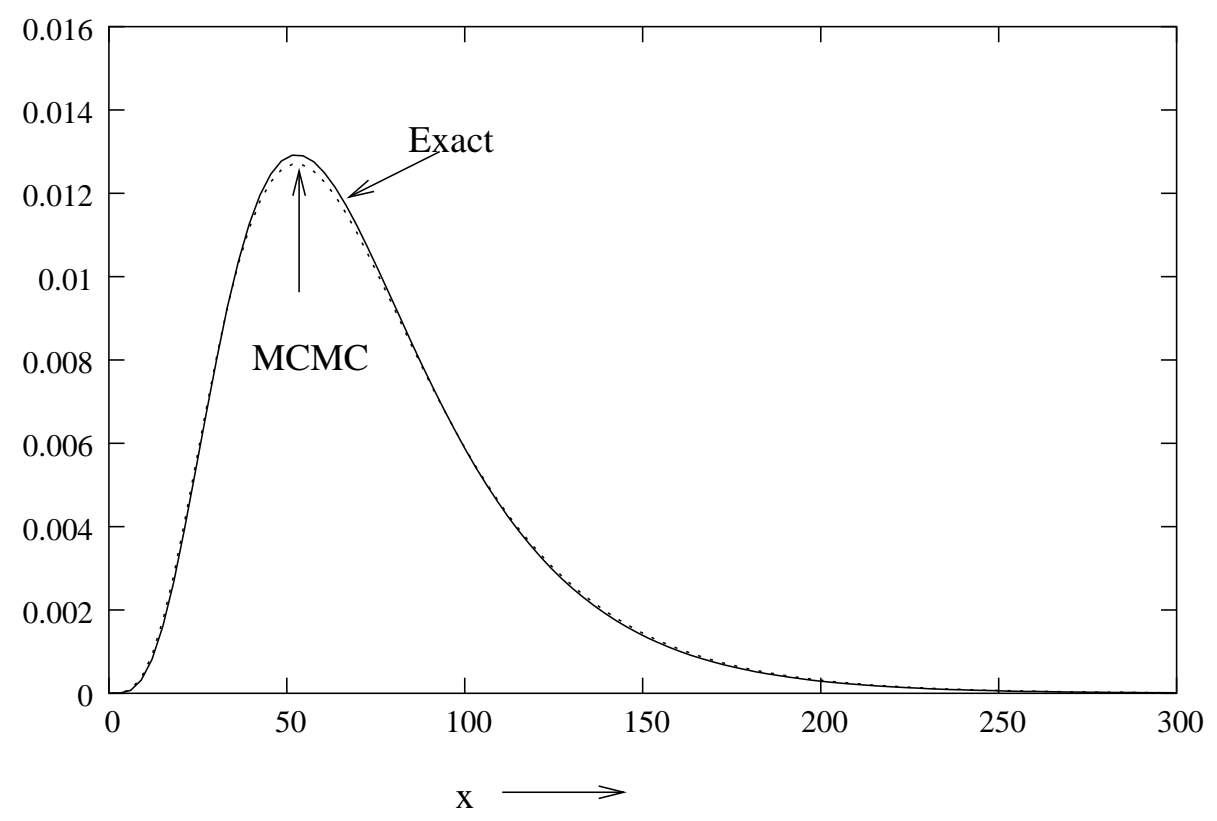

Figure 4: The density functions $f(x ; 5.3466,0.0318)$ and $f(x ; 5.1287,0.0311)$ are plotted.

\begin{tabular}{|c|c|c|}
\hline Methods & K-S Distance & $\mathrm{p}$-value \\
\hline MLE & 0.1058 & 0.9592 \\
Exact Bayes & 0.1218 & 0.8840 \\
App Bayes (MCMC) & 0.1251 & 0.8641 \\
\hline
\end{tabular}

Table 1: Kolmogorov-Smirnov distances and the corresponding p-values between the empirical distribution function and the fitted distribution functions

\section{Numerical Comparisons}

We have already observed in the previous section that the approximate Bayes estimators match quite well with the exact Bayes estimators. In this section our main aim is to compare the Bayes estimators with the classical maximum likelihood estimators. To make the comparison more meaningful, we assume the non-informative priors on both the shape and scale parameters. Since it is observed that approximate Bayes estimators work quite well even for small sample sizes and the exact Bayes estimators are quite difficult to compute 


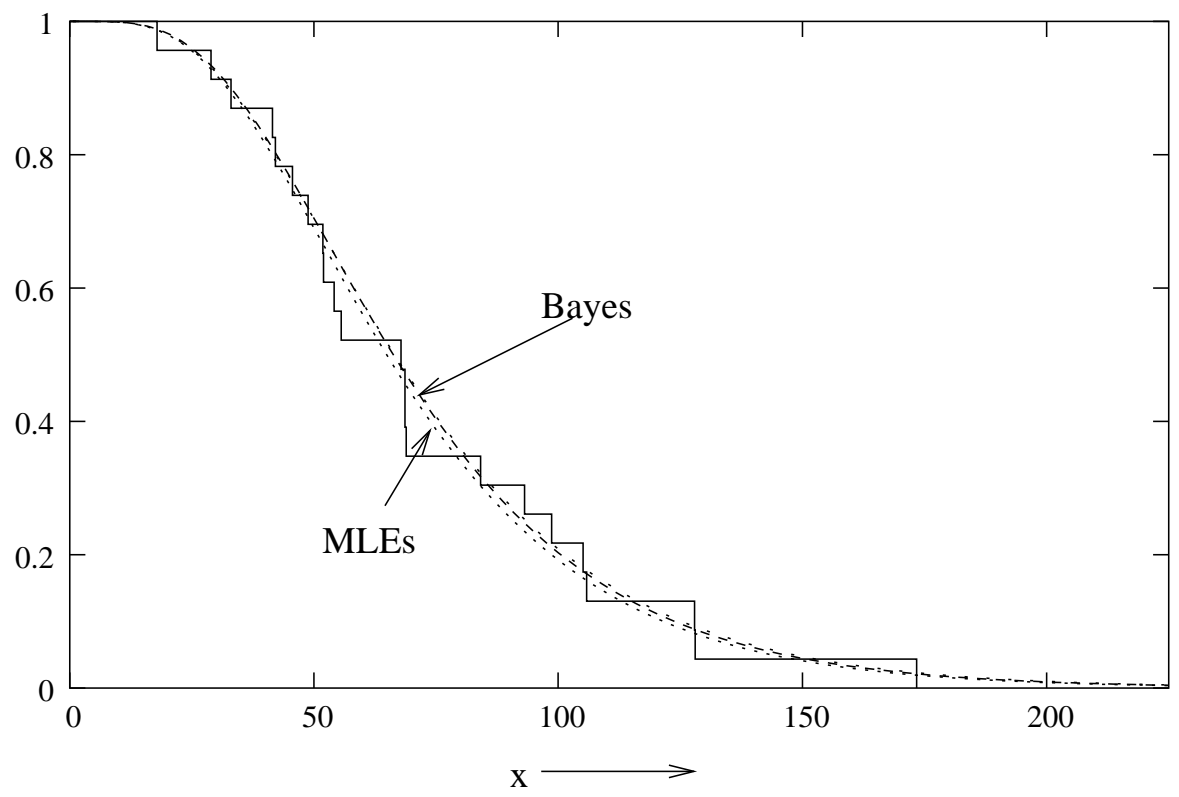

Figure 5: The estimated survival functions and the empirical survival function are plotted.

numerically, we compare the MLEs with the approximate Bayes estimates in terms of biases and square root of the mean squared errors (RMS) for different sample sizes and for different parameter values.

All the computations are performed at the Indian Institute of Technology Kanpur using Pentium IV processor. We use the random deviate generator RAN2 of Press et al. [15]. For each sample size we compute the MLEs of $\alpha$ and $\lambda$ and also the Bayes estimates using Lindley's approximation. We replicate the process 1000 times and compute the average estimates (AEs) and the RMS. The results are reported in Tables 2 and 3.

Some of the points are quite clear from the numerical results. As expected it is observed that the performances of all estimators become better when the sample size increases. It is also observed that both in terms of biases and RMS, for large sample sizes the Bayes estimates and the MLEs become closer. The Bayes estimates of $\lambda$ perform marginally better than the MLEs in terms of biases and RMS for all cases considered. In general the Bayes estimates of $\alpha$ perform better than the MLEs for $\alpha \leq 1$ and for $\alpha>1$ it is the other way. 
Table 2: Average relative estimates and the square root of the mean squared errors for the MLEs and approximate Bayes estimates of $\alpha$ when $\lambda$ is unknown.*

\begin{tabular}{|c|c|c|c|c|c|c|c|}
\hline$n$ & $\begin{array}{c}\alpha \rightarrow \\
\text { Methods } \downarrow\end{array}$ & 0.75 & 1.00 & 1.50 & 2.00 & 2.50 & 3.00 \\
\hline 15 & MLE & 1.2257 & 1.2541 & 1.2968 & 1.3642 & 1.4076 & 1.3999 \\
& & $(0.5842)$ & $(0.6583)$ & $(0.7393)$ & $(0.8967)$ & $(1.1311)$ & $(1.0312)$ \\
& ABAYES & 1.1971 & 1.2315 & 1.2864 & 1.3698 & 1.4323 & 1.4336 \\
& & $(0.5727)$ & $(0.6570)$ & $(0.7611)$ & $(0.9584)$ & $(1.3220)$ & $(1.1455)$ \\
\hline 25 & MLE & 1.1237 & 1.1363 & 1.1584 & 1.1773 & 1.1941 & 1.2094 \\
& & $(0.3527)$ & $(0.3817)$ & $(0.4331)$ & $(0.4782)$ & $(0.5191)$ & $(0.5567)$ \\
& \multirow{2}{*}{ ABAYES } & 1.1064 & 1.1215 & 1.1488 & 1.1733 & 1.1956 & 1.2165 \\
& & $(0.3448)$ & $(0.3761)$ & $(0.4333)$ & $(0.4851)$ & $(0.5333)$ & $(0.5787)$ \\
\hline 50 & MLE & 1.0732 & 1.0801 & 1.0718 & 1.0807 & 1.0850 & 1.0892 \\
& & $(0.2290)$ & $(0.2451)$ & $(0.2519)$ & $(0.2712)$ & $(0.2822)$ & $(0.2824)$ \\
& \multirow{2}{*}{ ABAYES } & 1.0647 & 1.0725 & 1.0664 & 1.0773 & 1.0836 & 1.0897 \\
& & $(0.2255)$ & $(0.2422)$ & $(0.2507)$ & $(0.2717)$ & $(0.2840)$ & $(0.2855)$ \\
\hline 75 & MLE & 1.0465 & 1.0507 & 1.0482 & 1.0512 & 1.0446 & 1.0675 \\
& & $(0.1682)$ & $(0.1790)$ & $(0.1940)$ & $(0.1984)$ & $(0.2037)$ & $(0.2201)$ \\
& \multirow{2}{*}{ ABAYES } & 1.0409 & 1.0457 & 1.0445 & 1.0487 & 1.0433 & 1.0675 \\
& & $(0.1662)$ & $(0.1773)$ & $(0.1932)$ & $(0.1984)$ & $(0.2044)$ & $(0.2214)$ \\
\hline
\end{tabular}

* In each box correspond to a method (MLE or ABAYES) and a $\alpha$, the first figure represents the average relative estimate of $\alpha$ and its RMS is reported within bracket immediately below. 
Table 3: Average relative estimates and the square root of the mean squared errors for the MLEs and approximate Bayes estimates of $\lambda$ when $\alpha$ is unknown.*

\begin{tabular}{|c|c|c|c|c|c|c|c|}
\hline$n$ & $\begin{array}{c}\alpha \rightarrow \\
\text { Methods } \downarrow\end{array}$ & 0.75 & 1.00 & 1.50 & 2.00 & 2.50 & 3.00 \\
\hline 15 & MLE & 1.2152 & 1.1877 & 1.1511 & 1.1526 & 1.1496 & 1.1417 \\
& & $(0.5561)$ & $(0.4900)$ & $(0.4027)$ & $(0.3784)$ & $(0.3800)$ & $(0.3601)$ \\
& ABAYES & 1.1735 & 1.1500 & 1.1216 & 1.1213 & 1.1197 & 1.1131 \\
& & $(0.5276)$ & $(0.4658)$ & $(0.3832)$ & $(0.3602)$ & $(0.3632)$ & $(0.3444)$ \\
\hline 25 & MLE & 1.1156 & 1.1018 & 1.0883 & 1.0815 & 1.0774 & 1.0747 \\
& & $(0.3495)$ & $(0.3140)$ & $(0.2777)$ & $(0.2587)$ & $(0.2468)$ & $(0.2386)$ \\
& \multirow{2}{*}{ ABAYES } & 1.0921 & 1.0802 & 1.0687 & 1.0632 & 1.0600 & 1.0579 \\
& & $(0.3368)$ & $(0.3031)$ & $(0.2685)$ & $(0.2504)$ & $(0.2392)$ & $(0.2314)$ \\
\hline 50 & MLE & 1.0693 & 1.0617 & 1.0458 & 1.0447 & 1.0382 & 1.0325 \\
& & $(0.2369)$ & $(0.2152)$ & $(0.1888)$ & $(0.1723)$ & $(0.1648)$ & $(0.1560)$ \\
& \multirow{2}{*}{ ABAYES } & 1.0579 & 1.0511 & 1.0362 & 1.0356 & 1.0295 & 1.0242 \\
& & $(0.2319)$ & $(0.2107)$ & $(0.1854)$ & $(0.1690)$ & $(0.1620)$ & $(0.1535)$ \\
\hline 75 & MLE & 1.0476 & 1.0422 & 1.0319 & 1.0253 & 1.0173 & 1.0314 \\
& & $(0.1846)$ & $(0.1681)$ & $(0.1484)$ & $(0.1340)$ & $(0.1301)$ & $(0.1238)$ \\
& \multirow{2}{*}{ ABAYES } & 1.0402 & 1.0353 & 1.0255 & 1.0193 & 1.0116 & 1.0259 \\
& & $(0.1817)$ & $(0.1656)$ & $(0.1464)$ & $(0.1324)$ & $(0.1289)$ & $(0.1220)$ \\
\hline
\end{tabular}

* In each box correspond to a method (MLE or ABAYES) and a $\alpha$, the first figure represents the average relative estimate of $\lambda$ and its RMS is reported within bracket immediately below. 


\section{EXtensions}

In this paper although we have discussed the Bayesian inference of the two-parameter generalized exponential distribution, but our method can be extended for many other cases also. We briefly describe them below.

\subsection{Proportional Reversed Hazard Model}

When the scale parameter $\lambda$ is known, the Bayes theory developed here, can be extended to the entire class of proportional reversed hazard models (see for example Di Crescenzo; $[1])$, i.e., the class of distribution functions $G(x ; \alpha)$, which can be expressed in the form $G(x ; \alpha)=(F(x))^{\alpha}$, for $\alpha>0$. Here $F(x)$ is a proper distribution function. Consider the following transformation $Y=-\ln (G(X))$, then $Y$ has an exponential distribution. If we

assume the gamma prior on $\alpha$ as given in (3), then it is the conjugate prior. It can be easily seen that, based on the random sample $\left\{x_{1}, \ldots, x_{n}\right\}$ from $G(x ; \alpha)$, the exact Bayes estimator of $\alpha$ under the squared error loss function is

$$
\frac{d+n}{c-\sum_{i=1}^{n} \ln F\left(x_{i}\right)} .
$$

In case of $G E$, when $\lambda$ is known it is a proportional reverse hazard class and the result (10) follows as a special case of (15).

\subsection{Exponentiated Weibull Model}

The exponentiated Weibull model, which was originally proposed by Mudholkar et al. [12] has the following probability density function;

$$
f(x ; \alpha, \lambda, \beta)=\alpha \lambda \beta\left(1-e^{-\lambda x^{\beta}}\right)^{\alpha-1} e^{-\lambda x^{\beta}} x^{\beta-1} ; \quad x>0
$$


Here $\alpha$ and $\lambda$ are same as before, $\beta>0$ is another shape parameter. To compute the Bayes estimates of the unknown parameters, $\{\alpha, \lambda, \beta\}$, it is assumed that $\lambda$ and $\alpha$ have the same priors as described in (2) and (3) respectively. The other shape parameter $\beta$ also has the gamma prior as

$$
\pi_{3}(\beta) \propto \beta^{v-1} e^{-u \beta} ; \quad \beta>0
$$

Here the hyper-parameters $u$ and $v$ are known and non-negative. Based on the observed sample $\left\{x_{1}, \ldots, x_{n}\right\}$, the posterior density function of $\alpha$ given $\lambda, \beta$ and data is

$$
l(\alpha \mid \lambda, \beta, \text { data }) \propto \alpha^{n+d-1} e^{-\alpha\left(c-\sum_{i=1}^{n} \ln \left(1-e^{-\lambda x_{i}^{\beta}}\right)\right)},
$$

which is a gamma probability density function with the shape and scale parameters as $n+d$ and $c-\sum_{i=1}^{n} \ln \left(1-e^{-\lambda x_{i}^{\beta}}\right)$ respectively.

The posterior density function of $\lambda$ given $\alpha, \beta$ and data is

$$
l(\lambda \mid \alpha, \beta, \text { data }) \propto \lambda^{b+n-1} e^{-\lambda\left(a+\sum_{i=1}^{n} x_{i}^{\beta}\right)} \prod_{i=1}^{n}\left(1-e^{-\lambda x_{i}^{\beta}}\right)^{\alpha-1} .
$$

It has the same form as (13), except $x_{i}$ is replaced by $x_{i}^{\beta}$. Therefore, the same method as (13), can be applied here also to generate samples from (19).

The posterior density function of $\beta$ given $\alpha, \lambda$ and data is

$$
\begin{aligned}
l(\beta \mid \alpha, \lambda, \text { data }) \propto & \beta^{n+v-1} \prod_{i=1}^{n}\left(1-e^{-\lambda x_{(i)}^{\beta}}\right)^{\alpha-1} e^{-\lambda \sum_{i=1}^{n} x_{(i)}^{\beta}} \prod_{i=1}^{n} x_{(i)}^{\beta-1} e^{-u \beta} \\
\propto & \left(1-e^{-\lambda x_{(1)}^{\beta}}\right)^{\alpha-1} e^{-\lambda x_{(1)}^{\beta}} \\
& \times\left[\beta^{n+v-1} e^{-u \beta} \prod_{i=1}^{n} x_{(i)}^{\beta-1} \prod_{i=2}^{n}\left(1-e^{-\lambda x_{(i)}^{\beta}}\right)^{\alpha-1} e^{-\lambda \sum_{i=2}^{n} x_{(i)}^{\beta}}\right]
\end{aligned}
$$

here as before, $x_{(1)}<\ldots<x_{(n)}$ are the ordered $x_{i}$ 's. Observe that the function within [.] is a continuous function in $\beta$. It vanishes as $\beta$ approaches 0 or $\infty$. It implies that the function has a finite maximum. Therefore with the help of the acceptance rejection principle again, the generation from (20) can be performed using the GE generator. Now using similar MCMC 
method as described in subsection 2.2, the posterior samples $\alpha, \lambda$ and $\beta$ can be generated and in turn the Bayes estimates and the corresponding credible intervals can be easily obtained. It should be mentioned that recently, Singh, Gupta and Upadhyay [21], Nassar and Eissa [13], Soliman, Abd Ellah and Sultan [22], Lee, Kim and Jung [10] and Ren, Sun and Dey [19] also consider the Bayes estimations of the parameters of some related models, but their approaches are quite different than ours.

\section{Conclusions}

In this paper we consider the Bayes estimation of the unknown parameters of the generalized exponential distribution. We assume the gamma priors on the unknown parameters and provide the Bayes estimators under the assumptions of squared error loss functions. It is observed that the Bayes estimators can not be obtained in explicit forms and they can be obtained using the numerical integration. Because of that we have used Lindley's approximation and it is observed that the approximation works very well. We have compared using Monte Carlo simulation between the performances of the MLEs and approximate Bayes estimators under the assumptions of non-informative priors and it is observed that the performances are quite similar.

We have also used MCMC technique to generate posterior sample and it is observed that the histograms of the generated posteriors match quite well with the theoretical posterior density functions. Since we have an effective MCMC technique we can use any other loss function also, for example, LINEX loss function or absolute error loss function. Moreover, we have seen that our method can be easily extended for exponentiated Weibull distribution also. Finally we should mention that, although we have used gamma priors on the shape parameter, but our method can be used for a more general class of priors also, for example priors with log-concave density functions. Choosing the proper priors is a challenging 
problem. More work is needed in that direction.

\section{ACKNOWLEDGEMENTS:}

The authors would like to thank two referees and one associate editor for their very constructive comments. The authors would also like to thank the Co-editor Professor J.C. Lee for his encouragements.

\section{APPENDIX:}

For the two parameter case, using the notation $\left(\lambda_{1}, \lambda_{2}\right)=(\alpha, \lambda)$, the Lindley's approximation can be written as follows;

$$
\hat{g}=g\left(\hat{\lambda}_{1}, \hat{\lambda}_{2}\right)+\frac{1}{2}\left[A+l_{30} B_{12}+l_{03} B_{21}+l_{21} C_{12}+l_{12} C_{21}\right]+p_{1} A_{12}+p_{2} A_{21},
$$

where

$$
\begin{gathered}
A=\sum_{i=1}^{2} \sum_{j=1}^{2} w_{i j} \tau_{i j}, \quad l_{i j}=\frac{\partial^{i+j} L\left(\lambda_{1}, \lambda_{2}\right)}{\partial \lambda_{1}^{i} \partial \lambda_{2}^{j}} ; \quad i, j=0,1,2,3, \quad i+j=3, \\
p_{i}=\frac{\partial p}{\partial \lambda_{i}}, \quad w_{i}=\frac{\partial g}{\partial \lambda_{i}}, \quad w_{i j}=\frac{\partial^{2} g}{\partial \lambda_{i} \partial \lambda_{j}}, \quad p=\ln \pi\left(\lambda_{1}, \lambda_{2}\right), \quad A_{i j}=w_{i} \tau_{i i}+w_{j} \tau_{j i}, \\
B_{i j}=\left(w_{i} \tau_{i i}+w_{j} \tau_{i j}\right) \tau_{i i}, \quad C_{i j}=3 w_{i} \tau_{i i} \tau_{i j}+w_{j}\left(\tau_{i i} \tau_{j j}+2 \tau_{i j}^{2}\right),
\end{gathered}
$$

here $L(.,$.$) is the log-likelihood function of the observed data, \pi\left(\lambda_{1}, \lambda_{2}\right)$ is the joint prior density function of $\left(\lambda_{1}, \lambda_{2}\right), \tau_{i j}$ is the $(i, j)$-th element of the inverse of the Fisher information

matrix. Moreover, $\hat{\lambda}_{1}$ and $\hat{\lambda}_{2}$ are the MLEs of $\lambda_{1}$ and $\lambda_{2}$ respectively and all the quantities are evaluated at $\left(\hat{\lambda}_{1}, \hat{\lambda}_{2}\right)$.

Now we have

$$
L(\alpha, \lambda)=n \ln \alpha+n \ln \lambda+(\alpha-1) \sum_{i=1}^{n} \ln \left(1-e^{-\lambda x_{i}}\right)-\lambda \sum_{i=1}^{n} x_{i} .
$$


Therefore, we obtain

$l_{30}=\frac{2 n}{\hat{\alpha}^{3}}, \quad l_{03}=\frac{2 n}{\hat{\lambda}^{3}}+(\hat{\alpha}-1) \sum_{i=1}^{n} \frac{x_{i}^{3} e^{-\hat{\lambda} x_{i}}\left(1+e^{-\hat{\lambda} x_{i}}\right)}{\left(1-e^{-\hat{\lambda} x_{i}}\right)^{3}}, \quad l_{12}=-\sum_{i=1}^{n} \frac{x_{i}^{2} e^{-\hat{\lambda} x_{i}}}{\left(1-e^{-\hat{\lambda} x_{i}}\right)^{2}}, \quad l_{21}=0$,

and $U, V$ and $W$ same as defined in (9). Now when $g(\alpha, \lambda)=\alpha$, then

$$
w_{1}=1, \quad w_{2}=0, \quad w_{i j}=0, \quad i, j=1,2,
$$

therefore,

$A=0, \quad B_{12}=\tau_{11}^{2}, \quad B_{21}=\tau_{21} \tau_{22}, \quad C_{12}=3 \tau_{11} \tau_{12}, \quad C_{21}=\left(\tau_{22} \tau_{11}+2 \tau_{21}^{2}\right), \quad A_{12}=\tau_{11}, \quad A_{21}=\tau_{12}$.

Now the first part of Lindley's approximation follows by using

$$
p_{1}=\frac{d-1}{\alpha}-c \quad \text { and } \quad p_{2}=\frac{b-1}{\lambda}-a .
$$

For the second part, note that $g(\alpha, \lambda)=\lambda$, then

$$
w_{1}=0, \quad w_{2}=1, \quad w_{i j}=0, \quad i, j=1,2,
$$

and

$A=0, \quad B_{12}=\tau_{12} \tau_{11}, \quad B_{21}=\tau_{22}^{2}, \quad C_{12}=\tau_{11} \tau_{22}+2 \tau_{12}^{2}, \quad C_{21}=3 \tau_{22} \tau_{21}, \quad A_{12}=\tau_{21}, \quad A_{21}=\tau_{22}$,

therefore the second part follows immediately.

\section{References}

[1] Di Crescenzo, A. (2000), "Some results on the proportional reversed hazards model", Statistics and Probability Letters, vol. 50, 313 - 321.

[2] Basu, S., Basu, A. P. and Mukhopadhyay, C. (1999), "Bayesian analysis for masked system failure data using non-identical Weibull models", Journal of the Statistical Planning and Inference, vol. 78, 255275. 
[3] Devroye, L. (1986), Nonuniform Random Variate Generation, Springer-Verlag, New York.

[4] Gupta, R. D. and Kundu, D. (1999). "Generalized exponential distributions", Australian and New Zealand Journal of Statistics, vol. 41, 173 - 188.

[5] Gupta, R. D. and Kundu, D. (2001), "Generalized exponential distributions: different methods of estimation", Journal of Statistical Computation and Simulation. vol. 69,315 - 338 .

[6] Gupta, R.D. and Kundu, D. (2007), "Generalized exponential distribution: existing results and some recent developments", Journal of Statistical Planning and Inference, doi: 10.1016/j.jspi.2007.03.030

[7] Jaheen, Z. F. (2004), "Empirical Bayes inference for generalized exponential distribution based on records", Communications in Statistics - Theory and Methods, vol. 33 , no. $8,1851-1861$.

[8] Kundu D and Gupta R.D. (2007), “ A convenient way of generating gamma random variables using generalized exponential distribution", Computational Statistics and Data Analysis, vol. 51, 2796-2802.

[9] Lawless, J, F. (1982), Statistical Models and Methods for Lifetime Data, John Wiley and Sons, New York.

[10] Lee J, Kim J, Jung S.H. (2007), "Bayesian analysis of paired survival data using a bivariate exponential distribution", Lifetime Data Analysis, vol. 13, 119-137.

[11] Lindley, D. V. (1980), "Approximate Bayesian method", Trabajos de Estadistica, vol. $31,223-237$. 
[12] Mudholkar, G.S. and Srivastava, D.K. (1993), "Exponentiated Weibull family for analyzing bathtub failure data", IEEE Trans. Reliability, vol. 42, 299302.

[13] Nassar, M. M. and Eissa, F. L. (2004), "Bayesian estimation for the exponentiated Weibull model", Communications in Statistics - Theory and Methods, vol. 33, no. $10,2343-2362$.

[14] Press, S. J. (2001), The Subjectivity of Scientists and the Bayesian Approach, Wiley, New York.

[15] Press, W. H., Teukolsky, S. A., Vellerling, W. T. and Flannery, B. P. (1994), Numerical Recipes in FORTRAN, 2nd. edition, Cambridge University Press, Cambridge.

[16] Raqab, M. Z. (2002), "Inferences for generalized exponential distribution based on record statistics", Journal of Statistical Planning and Inference, vol. 104, 339 - 350.

[17] Raqab, M. Z. and Ahsanullah, M. (2001), "Estimation of the location and scale parameters of generalized exponential distribution based on order statistics", Journal of Statistical Computation and Simulation, vol. 69, 109 - 124.

[18] Raqab, M.Z. and Madi, M.T. (2005), "Bayesian inference for the generalized exponential distribution", Journal of Statistical Computation and Simulation, vol. 75, no. $10,841-852$.

[19] Ren C,R., Sun D.C., Dey D.K. (2006), "Bayesian and frequentist estimation and prediction for exponential distributions" Journal of Statistical Planning and Inference, vol. 136, 2873-2897.

[20] Sarhan A.M. (2007), "Analysis of incomplete, censored data in competing risks models with generalized exponential distributions", IEEE Transactions on Reliability vol. 56, 132-138. 
[21] Singh, U., Gupta, P. K. and Upadhyay, S. K. (2005), "Estimation of parameters for exponentiated-Weibull family under type-II censoring scheme", Computational Statistics and Data Analysis, vol. 48, 509 - 523.

[22] Soliman A.A., Abd Ellah A.H., Sultan K.S. (2006), "Comparison of estimates using record statistics from Weibull model: Bayesian and non-Bayesian approaches", Computational Statistics and Data Analysis, vol. 51, 2065-2077.

[23] Zheng, G. (2002), "Fisher information matrix in type -II censored data from exponentiated exponential family", Biometrical Journal, vol. 44, 353 - 357. 